\title{
Effect of Dietary SID Methionine on Growth Performance, Carcass and Meat Quality, Myogenic Gene Expression in Fast and Slow Growing Coloured Chickens
}

\author{
Ho Le Quynh Chau, Than Thi Thanh Tra, Duong Thi Huong, Du Thanh Hang, Le Thi Thu \\ Hang, Vo Thi Minh Tam, Dinh Van Dung*, Ho Trung Thong
}

Faculty of Animal Sciences and Veterinary Medicine, University of Agriculture and Forestry, Hue University, Vietnam.

\begin{abstract}
The study was conducted to determine the effects of different levels of dietary SID methionine on growth performance, carcass yield, meat quality, myogenic gene expression of two coloured chicken groups. A total of 480 one-day-old chicks (240 Ri hybrid and 240 Luong Phuong) of uniform body weight was divided into a $2 \times 3$ factorial arrangement of treatments with four replicate cages of 20 chicks per treatment. Two factors include 2 chicken groups (Ri hybrid-slow-growing strain and Luong Phuong-fast-growing strain) and three dietary SID methionine concentration (low, adequate, high). The methionine levels were set up by the recommendation of Evonik (2010) for coloured chickens. At 12 weeks of age, 8 male and 8 female chicks per treatment were randomly chosen for the carcass yield, meat quality, and myogenic gene expression evaluation (myostatin, myf5, MEF2B). The results showed that the higher level of dietary SID methionine than that of Evonik recommendation (2010) improved growth performance in slow-growing colored chickens but had no effect on body weight gain in fast-growing strain. The increasing breast muscle yield and decreasing abdominal fat were observed in the fast-growing chicks fed high dietary SID methionine. The improvements in breast muscle of the fast-growing colored strain was reflected by increased mRNA expression of Myf5 and MEF2B but decreased expression of myostatin.
\end{abstract}

Keywords | Carcass, Fast-growing colored chickens, Methionine, Myogenic gene expression, Slow-growing colored chickens

Received | November 01, 2021; Accepted | December 21, 2021; Published | January 15, 2022

*Correspondence | Dinh Van Dung, Faculty of Animal Sciences and Veterinary Medicine, University of Agriculture and Forestry, Hue University, Vietnam; Email: dinhvandung@hueuni.edu.vn

Citation | Chau HLQ, Tra TTT, Huong DT, Hang DT, Hang LTT, Tam VTM, Dung DV, Thong HT (2022). Effect of dietary SID methionine on growth performance, carcass and meat quality, myogenic gene expression in fast and slow growing coloured chickens. Adv. Anim. Vet. Sci. 10(3): 529-536. DOI | http://dx.doi.org/10.17582/journal.aavs/2022/10.3.529.536

ISSN (Online) | 2307-8316

\section{INTRODUCTION}

$\mathrm{M}$ ethionine (Met) is usually the first limiting amino acid in most of the practical diets for broiler chicken (Ravindran and Bryden, 1999). Met is also an essential component in protein metabolism, provides methyl groups for choline and betaine synthesis (Corzo et al., 2006). Methionine also effects on lipid metabolism by stimulating the oxidative catabolism of fatty acids via carnitine synthesis (Nukreaw et al., 2011). Many studies have been reported the regulatory effects of Met on growth performance and breast meat yield in broilers (Hickling et al., 1990; Ahmed and Abbas, 2011; Bouyeh and Gevorgyan, 2011).Therefore, it is important to provide adequate Met to optimize broiler growth and production (Wen et al., 2017).

The Met contents given in the databases are generally minimum levels that satisfy general productive activities of broilers without strain being considered (Wen et al., 2017). Meanwhile, the different chicken groups may have differed Met requirement. Therefore, the responses of growth performance and productivity to dietary Met status may differ between strains (Wen et al., 2017).

The family of myogenic regulatory factors including myogenic differentiation factor 1 (myoD1), myogenic factor 5 (myf5), myogenin, and muscle regulatory factor 4 (MRF4), as well as the myocyte enhancer factor 2 (MEF2) 
family of transcription factors (MEF2A, B, C, and D), are known to be key regulators of skeletal muscle development (Hennebry et al., 2009; Townley-Tilson et al., 2010). The studies reported the expression of these factors affected by nutrition (essiential amino acids, cholecalciferol) in humans (Drummond et al., 2009) and animals (Alami-Durante et al., 2011). Myostatin is a negative regulator of skeletal muscle growth during the development and in the adult, and myostatin inhibition causes increased muscle mass (Wang and McPherron, 2012). Wen et al. (2014) also indicated that the increase in breast muscle growth in broilers fed + Met diets reflected the expected expression of myostatin, Myf5, and MEF2B genes. However, until now, little is known about the effects of Met on the expressions of these myogenic genes. Therefore, the objective of this study was to evaluate the effects of dietary SID Met concentrations on growth performance, breast and thigh muscle yields, meat quality, and the expression of these myogenic genes in fast- and slow-growing coloured chicken groups.

\section{MATERIALS AND METHODS}

\section{ANIMALS AND EXPERIMENTAL DESIGNS}

The Luong Phuong chicken was selected as the fast-growing broiler strain (FG), and Ri hybrid chick (1/4 Luong Phuong and $3 / 4 \mathrm{Ri}$ ) was selected as the slow-growing broiler strain (SG). A total of 240 chicks from each strain were obtained from National Institute of Animal Sciences, Vietnam and raised from 0 to 12 weeks of age. At 12 weeks of age, these broiler strains were reached to slaughter-weight. The chickens were divided into a $2 \times 3$ factorial arrangement of treatments with four replicate cages of twenty chicks per treatment unit. Vaccination against Newcastle was done at $1^{\text {st }}$ and $17^{\text {th }}$ day of age, while vaccination for infectious bursal disease followed at $6^{\text {th }}$ and $13^{\text {th }}$ day of age, and vaccination against Avian influenza infection was done at $15^{\text {th }}$ day of age.

The experimental diets were formulated based on corn, soybean meal, full fat soybean and fish meal using analyzed ingredient amino acid contents and published SID coefficients (Evonik, 2010). Three diets with low (0.44, $0.35,0.31,0.25)$, adequate $(0.52,0.43,0.39,0.33)$ and high $(0.60,0.51,0.47,0.41)$ SID methionine concentrations compared to SID methionine level recommended by Evonik (2010) for coloured chickens during 1-14, 15-30, $31-45$, and from 46 days of age, respectively (Table 1 ). The adequate diets were formulated to meet SID methionine requirement of the color chickens according to Evonik's (2010) at different ages. The low and the high SID methionine diets were formulated based on the adequate level $\pm 0.08 \%$.

\section{FEEDING AND MEASUREMENT}

Chickens were housed in $1.2 \times 2.5 \mathrm{~m}$ pens and allowed free access to feed and water. The environmental temperature ranged from $30-35^{\circ} \mathrm{C}$ during the experimental periods. The body weight of chicks and feed were recorded by biweekly to calculate body weight gain (BWG), feed intake (FI), FCR (feed conversion ratio) and PER (protein efficiency ratio). PER was calculated based on the average weight gain of a test chicken group divided by its protein intake. Mortality was also recorded.

\section{Sample collection}

At 12 weeks of age, 8 male and 8 female chicks per treatment were randomly selected and fasted for $12 \mathrm{~h}$, but water was offered. The individual weight of the birds was obtained and recorded just before killing by cervical dislocation. They were allowed to bleed for $5 \mathrm{~min}$, then put in pre-heated water and were plucked, scalded and washed. Evisceration and the cutting of the carcass into different parts were done manually. Weight of various parts was recorded using an electronic scale (Kern, Japan). The carcass yield was expressed as a percentage of the eviscerated carcass in the live weight.

\section{Carcass analysis}

The $\mathrm{pH}$ was measured $15 \mathrm{~min}(\mathrm{pH} 15)$ and $24 \mathrm{~h}(\mathrm{pH} 24)$ after slaughter on breast meat using a $\mathrm{pH}$ meter (HI99163, Hanna) calibrated at $\mathrm{pH} 4.0$ and 7.0. The $\mathrm{pH} 15$ and $\mathrm{pH} 24$ values were measured by directly inserting the glass electrode of the $\mathrm{pH}$-meter into the center of the breast meat. Breast meat colour was measured using a reflectance colorimeter (Minolta Chroma Meter CR-400) as described by Schilling et al. (2012). Three colour readings were taken, each measured in triplicate. Colour was measured using the $L^{*} a^{*} b^{*}$ colour scale, where $L^{*}$ represents degree of lightness $(0=$ black to $100=$ white $), a^{*}$ represents green $\left(a^{*}\right)$ to red $\left(+a^{*}\right)$, and $b^{*}$ represents blue $\left(b^{*}\right)$ to yellow $\left(+b^{*}\right)$. Colour for each sample was expressed in terms of CIE values for lightness $\left(\mathrm{L}^{*}\right)$, redness $\left(\mathrm{a}^{*}\right)$, and yellowness $\left(\mathrm{b}^{*}\right)$ (Schilling et al., 2012). Values for each colour parameter were averaged together.

The samples weighing about $50 \mathrm{~g}$ was obtained from the breast meat was used to assess drip loss, boiling loss as described by Schilling et al. (2012). Drip loss was measured by keeping samples suspended in covered plastic bag on sieved plastic racks for $24 \mathrm{~h}$ at $2-4^{\circ} \mathrm{C}$ and calculated as percentage of weight loss during storage. Boiling loss was measured by immersing cooking bags in hot water $\left(85^{\circ} \mathrm{C}\right)$ for approximately $20 \mathrm{~min}$ until an internal temperature of $80^{\circ} \mathrm{C}$ was reached. The bags were tempered at ambient temperature $\left(20^{\circ} \mathrm{C}\right)$ for $30 \mathrm{~min}$ before opening to drain the liquid from each bag. Each sample was patted dry with one paper towel and reweighed. Cooking loss was reported as a percentage and calculated as [(initial weight - final weight)/ initial weight] $\times 100$. 
Table 1: Ingredients and nutrient composition of experimental diets (as-fed basis).

\begin{tabular}{|c|c|c|c|c|c|c|c|c|c|c|c|c|}
\hline \multirow[t]{2}{*}{ Item } & \multicolumn{3}{|c|}{ 1-14 days old } & \multicolumn{3}{|c|}{ 15-30 days old } & \multicolumn{3}{|c|}{ 31-45 days old } & \multicolumn{3}{|c|}{ From 46 days old } \\
\hline & LM & $\mathrm{AM}$ & HM & LM & $\mathrm{AM}$ & HM & LM & $\mathrm{AM}$ & HM & LM & $\mathrm{AM}$ & HM \\
\hline \multicolumn{13}{|l|}{ Feed ingredients, $\%$} \\
\hline Corn, $7.65 \%$ CP & 48.70 & 48.62 & 48.54 & 54.66 & 54.58 & 54.50 & 56.67 & 56.59 & 56.51 & 62.34 & 62.26 & 62.17 \\
\hline SBM, $47.19 \%$ & 32.00 & 32.00 & 32.00 & 27.25 & 27.25 & 27.25 & 21.10 & 21.10 & 21.10 & 20.00 & 20.00 & 20.00 \\
\hline Fish meal, $64.60 \% \mathrm{CP}$ & - & - & - & 0.80 & 0.80 & 0.80 & 0.40 & 0.40 & 0.40 & - & - & - \\
\hline Full-fat soya, $36.40 \% \mathrm{CP}$ & 11.54 & 11.54 & 11.54 & 9.50 & 9.50 & 9.50 & 15.00 & 15.00 & 15.00 & 10.40 & 10.40 & 10.40 \\
\hline Oil & 4.08 & 4.08 & 4.08 & 4.50 & 4.50 & 4.50 & 4.05 & 4.05 & 4.05 & 4.70 & 4.70 & 4.70 \\
\hline DCP 19\% & 1.50 & 1.50 & 1.50 & 1.50 & 1.50 & 1.50 & 0.90 & 0.90 & 0.90 & 0.81 & 0.81 & 0.81 \\
\hline Limestone & 1.36 & 1.36 & 1.36 & 1.30 & 1.30 & 1.30 & 1.43 & 1.43 & 1.43 & 1.32 & 1.32 & 1.32 \\
\hline L-Lysine. $\mathrm{HCl}$ & 0.18 & 0.18 & 0.18 & 0.01 & 0.01 & 0.01 & - & - & - & - & - & - \\
\hline DL-Methionine & 0.14 & 0.22 & 0.30 & 0.07 & 0.15 & 0.23 & 0.04 & 0.12 & 0.20 & 0.00 & 0.08 & 0.17 \\
\hline L-Threonine & 0.10 & 0.10 & 0.10 & 0.01 & 0.01 & 0.01 & 0.01 & 0.01 & 0.01 & 0.03 & 0.03 & 0.03 \\
\hline Broiler $500^{1}$ & 0.20 & 0.20 & 0.20 & 0.20 & 0.20 & 0.20 & 0.20 & 0.20 & 0.20 & 0.20 & 0.20 & 0.20 \\
\hline Salt & 0.20 & 0.20 & 0.20 & 0.20 & 0.20 & 0.20 & 0.20 & 0.20 & 0.20 & 0.20 & 0.20 & 0.20 \\
\hline Total & 100 & 100 & 100 & 100 & 100 & 100 & 100 & 100 & 100 & 100 & 100 & 100 \\
\hline \multicolumn{13}{|c|}{ Calculated nutrient composition } \\
\hline DM, \% & 88.4 & 88.6 & 89.1 & 86.6 & 86.9 & 88.0 & 85.9 & 85.3 & 86.2 & 86.8 & 87.1 & 87.2 \\
\hline $\mathrm{ME}, \mathrm{kcal} / \mathrm{kg}$ & 3039 & 3037 & 3034 & 3106 & 3103 & 3100 & 3156 & 3153 & 3151 & 3201 & 3198 & 3195 \\
\hline $\mathrm{CP}, \%$ & 22.6 & 22.5 & 22.7 & 20.2 & 20.1 & 21.2 & 19.9 & 19.9 & 19.8 & 17.2 & 17.3 & 17.4 \\
\hline $\mathrm{EE}, \%$ & 7.21 & 7.53 & 7.39 & 7.75 & 7.12 & 7.88 & 6.47 & 6.41 & 6.49 & 7.69 & 7.51 & 7.91 \\
\hline $\mathrm{CF}, \%$ & 2.38 & 2.38 & 2.37 & 2.53 & 2.71 & 2.41 & 3.01 & 3.29 & 3.17 & 2.94 & 3.13 & 3.13 \\
\hline SID Lys, \% & 1.31 & 1.31 & 1.31 & 1.05 & 1.05 & 1.05 & 0.97 & 0.97 & 0.97 & 0.85 & 0.85 & 0.85 \\
\hline SID Met, \% & 0.44 & 0.52 & 0.60 & 0.35 & 0.43 & 0.51 & 0.31 & 0.39 & 0.47 & 0.25 & 0.33 & 0.41 \\
\hline SID Cys, \% & 0.30 & 0.30 & 0.30 & 0.28 & 0.28 & 0.28 & 0.27 & 0.27 & 0.27 & 0.25 & 0.25 & 0.25 \\
\hline SID Met+Cys, \% & 0.74 & 0.82 & 0.90 & 0.63 & 0.71 & 0.78 & 0.57 & 0.65 & 0.73 & 0.49 & 0.57 & 0.66 \\
\hline Calcium, \% & 1.00 & 1.00 & 1.00 & 1.00 & 1.00 & 1.00 & 0.90 & 0.90 & 0.90 & 0.80 & 0.80 & 0.80 \\
\hline Avail P, \% & 0.45 & 0.45 & 0.45 & 0.45 & 0.45 & 0.45 & 0.35 & 0.35 & 0.35 & 0.30 & 0.30 & 0.30 \\
\hline \multicolumn{13}{|l|}{ Ratio to SID Lys } \\
\hline SID Met, \% & 34 & 40 & 46 & 34 & 41 & 49 & 32 & 40 & 48 & 29 & 38 & 49 \\
\hline SID Thr, $\%$ & 56 & 62 & 68 & 60 & 67 & 75 & 59 & 67 & 75 & 58 & 67 & 78 \\
\hline SID Trp, \% & 64 & 64 & 64 & 65 & 65 & 65 & 66 & 66 & 66 & 71 & 71 & 71 \\
\hline SID Arg, \% & 19 & 19 & 19 & 21 & 21 & 21 & 22 & 22 & 22 & 22 & 22 & 22 \\
\hline SID Ile, $\%$ & 112 & 112 & 112 & 124 & 124 & 124 & 127 & 127 & 127 & 129 & 129 & 129 \\
\hline SID Leu, \% & 68 & 68 & 68 & 76 & 76 & 76 & 78 & 78 & 78 & 79 & 79 & 79 \\
\hline SID Val, \% & 131 & 131 & 131 & 152 & 152 & 152 & 158 & 158 & 158 & 168 & 168 & 168 \\
\hline SID His, \% & 73 & 73 & 73 & 82 & 82 & 82 & 84 & 84 & 84 & 87 & 87 & 87 \\
\hline SID Phe, \% & 43 & 43 & 43 & 48 & 48 & 48 & 50 & 50 & 50 & 52 & 52 & 52 \\
\hline
\end{tabular}

$11 \mathrm{~kg}$ contains 6,000,000 UI vitamin A; 17,000mg vitamin $\mathrm{E} ; 1,460 \mathrm{mg}$ vitamin $\mathrm{K}$; 1,500mg vitamin B1; 80mg biotin; 40,00044,000mg Zn; 90,000-98,000mg Mn. LM, low methionine; AM, adequate methionine, HM, high methionine.

Shear force was assessed using a Warner-Bratzler Shear force procedure described in Schilling et al. (2012). The samples used for cooking loss determinations were used for shear force determinations. Four to 6 adjacent $1 \mathrm{~cm}$ (width) $\times 1 \mathrm{~cm}$ (thickness) $\times 2 \mathrm{~cm}$ (length, parallel to the orientation of the muscle fibers) strips were cut from the cooked breast fillet, parallel to the direction of the muscle fibers. Each strip was sheared once, and the mean was calculated for each fillet. Samples were sheared perpendicular to the muscle fibers using WDS- 1 instrument with a cross head speed of $200 \mathrm{~mm} / \mathrm{min}$.

\section{Gene EXPRESSION ANALYSIS}

Atotalof 36 breastmuscle samples $(3$ male and 3 femalechicks per treatment) was collected for gene expression analysis. Briefly, total RNA of breast muscle samples was extracted with Total RNA Purification kit (Cat. \#17200, Norgen, Biotek, Canada) according to the manufacturer's protocol. 
Table 2: Primer sequences for real-time PCR primers.

\begin{tabular}{|c|c|c|c|}
\hline Gene & Gene bank ID & Primer sequence $\left(5^{\prime}-3^{\prime}\right)$ & Product size, bp \\
\hline \multirow[t]{2}{*}{ Myostatin } & NM_001001461 & GGGACGTTATTAAGCAGC & \multirow[t]{2}{*}{153} \\
\hline & & ACTCCGTAGGCATTGTGA & \\
\hline \multirow[t]{2}{*}{ Myf5 } & NM_001030363 & CTTCGAGGCGGGCTACTG & \multirow[t]{2}{*}{106} \\
\hline & & AGAGAGGCGGTCCACGAT & \\
\hline \multirow[t]{2}{*}{ MEF2B } & XM_430389 & CACAGCCTCACTGGTTTCCC & \multirow[t]{2}{*}{230} \\
\hline & & GACACCCGCTCTGACTTGATG & \\
\hline \multirow[t]{2}{*}{ GAPDH } & NM_204305 & AGAACATCATCCCAGCGTCC & \multirow[t]{2}{*}{133} \\
\hline & & CGGCAGGTCAGGTCAACAAC & \\
\hline
\end{tabular}

The purity and concentration of total RNA in each sample were determined through $260 / 280 \mathrm{~nm}$ absorbance measures using the NanoDrop spectrophotometer (Thermo Scientific, USA). A ratio of 1.9-2.1 is generally accepted as "pure" for RNA. The diluted RNA was reverse transcribed into cDNA with iScript ${ }^{\mathrm{TM}} \mathrm{cDNA}$ synthesis kit (Biorad, USA). The cDNA was quantified using Maxima SYBR Green/ROX qPCR Master mix kit (Thermo Scientific) on Real-time PCR QuantStudio 5 system (Thermo Fisher Scientific). Optimised cycling conditions were $95^{\circ} \mathrm{C}$ for $30 \mathrm{~s}$ followed by 40 cycles of $95^{\circ} \mathrm{C}$ for $5 \mathrm{~s}, 60^{\circ} \mathrm{C}$ for 31 $\mathrm{s}$, and final dissociation stage of $95^{\circ} \mathrm{C}$ for $15 \mathrm{~s}, 60^{\circ} \mathrm{C}$ for $1 \mathrm{~min}, 95^{\circ} \mathrm{C}$ for $15 \mathrm{~s}$ and $60^{\circ} \mathrm{C}$ for $15 \mathrm{~s}$. The geometric means of glyceraldehyde 3-phosphate dehydrogenase (GAPDH) was used to normalise the genes of interest as recommended. The primers sequences for GAPDH, MSTN, Myf5, MEF2B was synthesized according to Wen et al (2014). The amplified products also were presented in Table 2. Relative mRNA levels (arbitrary units) were calculated on the basis of PCR efficiency and threshold cycle $(\mathrm{Ct})$ values using the $2^{-\Delta \Delta \mathrm{Ct}}$ method (Livak and Schmittgen, 2001). The mRNA level of each target gene in the fast-growing chicks fed the AM diets was assigned a value of one.

\section{STATISTICAL ANALYSis}

Two-way ANOVA was performed to determine the main effects of strain and Met concentration and their interaction using the general linear model procedure of SPSS software (version 15.0; SPSS Inc.). The differences were considered to be significant at $\mathrm{P}<0.05$. Data are presented as means $(\mathrm{M})$ and pooled standard error of the means (SEM).

\section{RESULTS}

\section{GROWTH PERFORMANCE}

The effects of dietary SID methionine on growth performance of chickens were presented in Table 3. Higher body weight gain (BWG), feed intake (FI), crude protein intake (CPI), protein efficiency ratio (PER) and lower feed conversion ratio $(\mathrm{FCR})$ were observed $(\mathrm{P}<0.05)$ in the fast-growing chicks than in the slow-growing strain. There was no difference in these criteria between chicks fed low methionine (LM), adequate methionine (AM) or high methionine (HM) diets. Compared with the LM and AM diets, the HM diets increased $(\mathrm{P}<0.05) \mathrm{BWG}$ and PER, as well as decreased the FCR in slow-growing chickens. Meanwhile, the HM diets increased $(\mathrm{P}<0.05) \mathrm{CPI}$ in fast-growing strain compared with the LM diets. There was no effect of the SID methionine concentration, and the interaction between SID methionine level and strain on FI and CPI within each strain. Mortality was 3.2\% and not related to treatment.

Table 3: Effects of dietary SID methionine on growth performance of chickens from 0 to 12 weeks of age.

\begin{tabular}{|c|c|c|c|c|c|}
\hline Item & BWG, $\mathrm{g}$ & FI, $g$ & CPI, $g$ & FCR & PER \\
\hline \multicolumn{6}{|l|}{ Strain } \\
\hline SG & $1273.1^{\mathrm{b}}$ & $4464.2^{\mathrm{b}}$ & $937.6^{\mathrm{b}}$ & $3.51^{a}$ & $1.36^{\mathrm{b}}$ \\
\hline FG & $1808.8^{a}$ & $5847.9^{\mathrm{a}}$ & $1220.0^{\mathrm{a}}$ & $3.23^{\mathrm{b}}$ & $1.48^{\mathrm{a}}$ \\
\hline SEM & 14.87 & 12.20 & 2.91 & 0.04 & 0.02 \\
\hline \multicolumn{6}{|l|}{ Methionine } \\
\hline LM & 1508.0 & 5151.1 & 1075.7 & 3.45 & 1.39 \\
\hline $\mathrm{AM}$ & 1525.5 & 5144.8 & 1078.2 & 3.41 & 1.41 \\
\hline HM & 1589.4 & 5172.4 & 1082.5 & 3.26 & 1.47 \\
\hline SEM & 144.0 & 370.0 & 75.6 & 0.09 & 0.04 \\
\hline \multicolumn{6}{|l|}{ Treatment } \\
\hline $\mathrm{SG}+\mathrm{LM}$ & $1229.0^{c}$ & $4470.5^{\mathrm{b}}$ & $937.3^{c}$ & $3,64^{a}$ & $1,31^{\mathrm{b}}$ \\
\hline $\mathrm{SG}+\mathrm{AM}$ & $1238.6^{c}$ & $4453.3^{b}$ & $937.4^{\mathrm{c}}$ & $3.60^{\mathrm{a}}$ & $1.32^{\mathrm{b}}$ \\
\hline $\mathrm{SG}+\mathrm{HM}$ & $1351.8^{\mathrm{b}}$ & $4468.8^{\mathrm{b}}$ & $937.9^{c}$ & $3.31^{\mathrm{b}}$ & $1.44^{\mathrm{a}}$ \\
\hline $\mathrm{FG}+\mathrm{LM}$ & $1787.1^{\mathrm{a}}$ & $5831.6^{\mathrm{a}}$ & $1213.6^{\mathrm{b}}$ & $3.26^{\mathrm{b}}$ & $1.47^{\mathrm{a}}$ \\
\hline $\mathrm{FG}+\mathrm{AM}$ & $1812.4^{\mathrm{a}}$ & $5836.2^{a}$ & $1218.9^{\mathrm{ab}}$ & $3.22^{\mathrm{b}}$ & $1.49^{\mathrm{a}}$ \\
\hline $\mathrm{FG}+\mathrm{HM}$ & $1827.4^{\mathrm{a}}$ & $5876.0^{\mathrm{a}}$ & $1227.6^{a}$ & $3.22^{\mathrm{b}}$ & $1.49^{\mathrm{a}}$ \\
\hline SEM & 15.6 & 18.6 & 3.99 & 0.04 & 0.02 \\
\hline \multicolumn{6}{|l|}{$P$ value } \\
\hline Strain & $<0.01$ & $<0.01$ & $<0.01$ & $<0.01$ & $<0.01$ \\
\hline Methionine & 0.84 & 1.00 & 1.00 & 0.10 & 0.15 \\
\hline Strain $\times$ methionine & $<0.01$ & 0.25 & 0.06 & $<0.01$ & $<0.01$ \\
\hline
\end{tabular}

BWG, body weight gain; FI, feed intake; CPI, crude protein intake; FCR, feed conversion ratio; PER, protein efficiency ratio; $\mathrm{LM}$, low methionine diet; $\mathrm{AM}$, adequate methionine diet; HM, high methionine diet; SG, slow-growing strain; FG, fastgrowing strain. Results are presented as means and pooled SEM $(n=4)$. Mean values within a column with unlike superscript letters were significantly different $(\mathrm{P}<0.05)$. 
CARCASS YIELD AND MEAT QUALITY

Except relative thigh + drumstick and abdominal fat weight, the carcass composition and carcass yield obtained in fast-growing chicks were higher $(\mathrm{P}<0.05)$ than those in slow-growing strain (Table 4). Meanwhile, there was no difference in these criteria between chicks fed LM, AM or HM diets. Chickens fed the HM diets had higher $(\mathrm{P}<0.05)$ relative thigh + drumstick weight than the chicks fed LM and AM diets. The highest absolute and relative weight of breast muscle were observed in fast-growing chicks fed HM diet $(\mathrm{P}<0.05)$. Chickens fed HM diets had the lowest abdominal fat retention.
There was no significant difference $(\mathrm{P}>0.05)$ in the $\mathrm{pH}$ values, drip loss, and shear force between two strains (Table 5). The cooking loss of breast meat collected from fast-growing chicks was lower than slow-growing strain. However, the $\mathrm{pH}$ values and water loss of breast meat did not differ between chickens fed LM, AM and HM diets. The lowest total water loss of breast meat was observed in fast-growing chicks fed LM diet. Whereas, the greatest shear force was in slow-growing chickens fed HM diet. All samples demonstrated $L^{*}$ values less than 60 . There was no interaction between SID methionine and strain effects on $\mathrm{pH} 15$, drip loss and total loss of breast meat within each strain.

Table 4: Effects of dietary SID methionine on carcass characteristics of chickens at 12 weeks of age.

\begin{tabular}{|c|c|c|c|c|c|c|c|c|c|}
\hline \multirow[t]{2}{*}{ Item } & \multicolumn{5}{|c|}{ Carcass composition, $\mathbf{g}$} & \multicolumn{4}{|c|}{ Carcass yield, $\%$} \\
\hline & $\begin{array}{l}\text { Live } \\
\text { weight }\end{array}$ & $\begin{array}{l}\text { Carcass } \\
\text { weight }\end{array}$ & $\begin{array}{l}\text { Breast } \\
\text { weight }\end{array}$ & $\begin{array}{l}\text { Thigh and } \\
\text { drumstick weight }\end{array}$ & $\begin{array}{l}\text { Abdominal } \\
\text { fat }\end{array}$ & $\begin{array}{l}\text { Dressing } \\
\text { out }\end{array}$ & Breast & $\begin{array}{l}\text { Thigh and } \\
\text { drumstick }\end{array}$ & $\begin{array}{l}\text { Abdominal } \\
\text { fat }\end{array}$ \\
\hline \multicolumn{10}{|l|}{ Strain } \\
\hline $\mathrm{SG}$ & $1301.7^{b}$ & $886.8^{\mathrm{b}}$ & $155.8^{\mathrm{b}}$ & $200.4^{\mathrm{b}}$ & $6.81^{b}$ & $68.06^{\mathrm{b}}$ & $17.56^{\mathrm{b}}$ & 22.55 & 0.07 \\
\hline $\mathrm{FG}$ & $1846.8^{\mathrm{a}}$ & $1284.4^{\mathrm{a}}$ & $247.3^{\mathrm{a}}$ & $291.2^{\mathrm{a}}$ & $14.23^{\mathrm{a}}$ & $69.55^{\mathrm{a}}$ & $19.24^{\mathrm{a}}$ & 22.67 & 1.11 \\
\hline SEM & 17.0 & 15.3 & 3.3 & 4.0 & 2.84 & 0.40 & 0.33 & 0.29 & 0.21 \\
\hline \multicolumn{10}{|l|}{ Methionine } \\
\hline LM & 1542.1 & 1062.0 & 194.4 & 232.3 & $19.0^{\mathrm{a}}$ & 68.58 & 18.17 & $21.82^{\mathrm{b}}$ & $1.63^{\mathrm{a}}$ \\
\hline $\mathrm{AM}$ & 1559.2 & 1073.7 & 193.8 & 239.9 & $8.81^{\mathrm{b}}$ & 68.72 & 17.96 & $22.37^{\mathrm{b}}$ & $0.83^{\mathrm{b}}$ \\
\hline $\mathrm{HM}$ & 1621.5 & 1121.3 & 216.4 & 265.2 & $3.75^{\mathrm{b}}$ & 69.15 & 19.08 & $23.63^{\mathrm{a}}$ & $0.37^{\mathrm{b}}$ \\
\hline SEM & 117.6 & 86.2 & 20.0 & 20.1 & 2.97 & 0.53 & 0.52 & 0.40 & 0.20 \\
\hline \multicolumn{10}{|l|}{ Treatment } \\
\hline $\mathrm{SG}+\mathrm{LM}$ & $1260.5^{c}$ & $845.6^{c}$ & $148.90^{c}$ & $183.40^{\mathrm{d}}$ & $7.30^{c}$ & $67.07^{c}$ & $17.60^{\mathrm{b}}$ & $21.65^{b}$ & $0.86^{\mathrm{b}}$ \\
\hline $\mathrm{SG}+\mathrm{AM}$ & $1268.2^{c}$ & $862.5^{c}$ & $151.54^{c}$ & $193.96^{\mathrm{d}}$ & $7.60^{c}$ & $68.00^{\mathrm{bc}}$ & $17.55^{\mathrm{b}}$ & $22.50^{\mathrm{ab}}$ & $0.88^{b}$ \\
\hline $\mathrm{SG}+\mathrm{HM}$ & $1376.5^{b}$ & $952.3^{\mathrm{b}}$ & $166.83^{c}$ & $223.61^{\mathrm{c}}$ & $5.56^{c}$ & $69.19^{\mathrm{ab}}$ & $17.53^{\mathrm{b}}$ & $23.49^{a}$ & $0.58^{c}$ \\
\hline $\mathrm{FG}+\mathrm{LM}$ & $1823.7^{\mathrm{a}}$ & $1278.2^{\mathrm{a}}$ & $239.61^{b}$ & $280.99^{b}$ & $30.71^{\mathrm{a}}$ & $70.09^{a}$ & $18.74^{\mathrm{b}}$ & $21.98^{\mathrm{ab}}$ & $2.40^{\mathrm{a}}$ \\
\hline $\mathrm{FG}+\mathrm{AM}$ & $1850.2^{\mathrm{a}}$ & $1284.9^{a}$ & $235.99^{b}$ & $285.94^{\mathrm{ab}}$ & $10.02^{\mathrm{b}}$ & $69.45^{\mathrm{ab}}$ & $18.37^{\mathrm{b}}$ & $22.25^{\mathrm{ab}}$ & $0.78^{b}$ \\
\hline $\mathrm{FG}+\mathrm{HM}$ & $1866.5^{\mathrm{a}}$ & $1290.2^{\mathrm{a}}$ & $265.86^{\mathrm{a}}$ & $306.56^{a}$ & $1.94^{\mathrm{d}}$ & $69.12^{\mathrm{ab}}$ & $20.62^{\mathrm{a}}$ & $23.77^{\mathrm{a}}$ & $0.15^{\mathrm{d}}$ \\
\hline SEM & 23.4 & 19.0 & 6.22 & 7.91 & 0.72 & 0.49 & 0.46 & 0.59 & 0.06 \\
\hline \multicolumn{10}{|l|}{ Pvalue } \\
\hline Strain & $<0.01$ & $<0.01$ & $<0.01$ & $<0.01$ & $<0.01$ & $<0.01$ & $<0.01$ & 0.68 & 0.13 \\
\hline Methionine & 0.78 & 0.77 & 0.50 & 0.25 & $<0.01$ & 0.53 & 0.10 & $<0.01$ & $<0.01$ \\
\hline Strain $\times$ methionine & 0.23 & 0.01 & $<0.01$ & $<0.01$ & $<0.01$ & $<0.01$ & $<0.01$ & $<0.01$ & $<0.01$ \\
\hline
\end{tabular}

Results are presented as means and pooled SEM $(\mathrm{n}=16)$. Mean values within a column with unlike superscript letters were significantly different $(\mathrm{P}<0.05)$.

\section{GENE EXPRESSION}

The fast-growing chickens had higher $(\mathrm{P}<0.05)$ mRNA expression of Myf5 and MEF2B, but lower mRNA expression of MSTN in breast muscle than the slowgrowing chickens (Table 6). Compared with the LM and AM diets, the HM diets increased $(\mathrm{P}<0.05)$ mRNA levels of MEF2B, and decreased MSTN mRNA level in both strains. There was no significantly difference in mRNA expression of Myf5 between chicks fed LM, AM and HM diets. The greatest $m R N A$ expression of Myf5 and MEF2B, and the lowest MSTN mRNA level were shown in fastgrowing chicks fed HM diets. Effect of the interaction between SID methionine level and strain was observed on mRNA expression of MSTN, Myf5 and MEF2B. 
Table 5: Effects of dietary SID methionine on meat quality of chickens at 12 weeks of age.

\begin{tabular}{|c|c|c|c|c|c|c|c|c|c|}
\hline \multirow[t]{2}{*}{ Item } & \multicolumn{2}{|c|}{ pH value, unit } & \multicolumn{2}{|c|}{ Water loss, \% } & \multicolumn{4}{|c|}{ Color coordinate } & \multirow{2}{*}{$\begin{array}{l}\text { Shear } \\
\text { force, } N\end{array}$} \\
\hline & pH15 & $\mathrm{pH} 24$ & Drip loss & Cooking loss & Total loss & L*(Lightness) & $\mathrm{a}^{*}$ (Redness) & $\mathrm{b}^{*}$ (Yellowness) & \\
\hline \multicolumn{10}{|l|}{ Strain } \\
\hline SG & 5.80 & 5.38 & 0.95 & $16.66^{\mathrm{a}}$ & $17.61^{\mathrm{a}}$ & $53.81^{b}$ & 2.87 & $9.11^{\mathrm{a}}$ & 26.10 \\
\hline FG & 5.72 & 5.34 & 0.89 & $14.21^{b}$ & $15.09^{b}$ & $56.59^{a}$ & 2.98 & $7.56^{b}$ & 24.40 \\
\hline SEM & 0.05 & 0.05 & 0.08 & 0.66 & 0.71 & 0.36 & 0.12 & 0.41 & 1.16 \\
\hline \multicolumn{10}{|l|}{ Methionine } \\
\hline LM & 5.74 & 5.40 & 0.89 & 16.27 & 17.16 & $53.24^{\mathrm{b}}$ & $3.54^{\mathrm{a}}$ & $7.88^{b}$ & $26.43^{a}$ \\
\hline $\mathrm{AM}$ & 5.81 & 5.37 & 0.88 & 14.85 & 15.73 & $55.53^{\mathrm{a}}$ & $2.95^{\mathrm{b}}$ & $9.07^{a}$ & $22.87^{b}$ \\
\hline HM & 5.73 & 5.32 & 0.99 & 15.17 & 16.16 & $56.83^{\mathrm{a}}$ & $2.28^{c}$ & $8.13^{\mathrm{ab}}$ & $26.44^{\mathrm{a}}$ \\
\hline SEM & 0.06 & 0.05 & 0.10 & 0.81 & 0.86 & 0.73 & 0.14 & 0.50 & 1.14 \\
\hline \multicolumn{10}{|l|}{ Treatment } \\
\hline $\mathrm{SG}+\mathrm{LM}$ & 5.78 & 5.46 & 1.05 & $19.33^{\mathrm{a}}$ & $20.38^{a}$ & $52.26^{\mathrm{d}}$ & $3.62^{\mathrm{a}}$ & $7.61^{c}$ & $25.34^{\mathrm{c}}$ \\
\hline $\mathrm{SG}+\mathrm{AM}$ & 5.89 & 5.40 & 0.91 & $15.02^{\mathrm{b}}$ & $15.93^{\mathrm{b}}$ & $53.34^{\mathrm{cd}}$ & $2.73^{b c}$ & $10.85^{\mathrm{a}}$ & $22.37^{\mathrm{d}}$ \\
\hline SG + HM & 5.72 & 5.28 & 0.89 & $15.63^{b}$ & $16.51^{\mathrm{b}}$ & $55.83^{\mathrm{b}}$ & $2.25^{\mathrm{c}}$ & $8.86^{b}$ & $30.59^{a}$ \\
\hline $\mathrm{FG}+\mathrm{LM}$ & 5.69 & 5.34 & 0.72 & $13.22^{\mathrm{c}}$ & $13.94^{\mathrm{c}}$ & $54.22^{c}$ & $3.45^{\mathrm{a}}$ & $8.00^{c}$ & $27.53^{\mathrm{b}}$ \\
\hline $\mathrm{FG}+\mathrm{AM}$ & 5.73 & 5.33 & 0.85 & $14.68^{b}$ & $15.52^{\mathrm{b}}$ & $57.73^{a}$ & $3.17^{\mathrm{ab}}$ & $7.29^{c}$ & $23.37^{\mathrm{d}}$ \\
\hline $\mathrm{FG}+\mathrm{HM}$ & 5.73 & 5.35 & 1.09 & $14.72^{\mathrm{b}}$ & $15.81^{\mathrm{b}}$ & $57.84^{\mathrm{a}}$ & $2.31^{c}$ & $7.40^{c}$ & $22.30^{\mathrm{d}}$ \\
\hline SEM & 0.08 & 0.07 & 0.14 & 0.41 & 0.47 & 0.53 & 0.20 & 0.25 & 0.61 \\
\hline \multicolumn{10}{|l|}{ Pvalue } \\
\hline Strain & 0.09 & 0.40 & 0.46 & $<0.01$ & $<0.01$ & $<0.01$ & 0.37 & $<0.01$ & 0.16 \\
\hline Methionine & 0.33 & 0.31 & 0.57 & 0.20 & 0.23 & $<0.01$ & $<0.01$ & $<0.05$ & $<0.01$ \\
\hline $\begin{array}{l}\text { Strain } \times \text { me- } \\
\text { thionine }\end{array}$ & 0.54 & 0.02 & 0.81 & 0.04 & 0.07 & $<0.01$ & 0.02 & $<0.01$ & $<0.01$ \\
\hline
\end{tabular}

Results are presented as means and pooled SEM ( $\mathrm{n}=16)$. Mean values within a column with unlike superscript letters were significantly different $(\mathrm{P}<0.05)$

Table 6: Effects of dietary SID methionine on relative mRNA levels in beast muscle of chickens at 12 weeks of age.

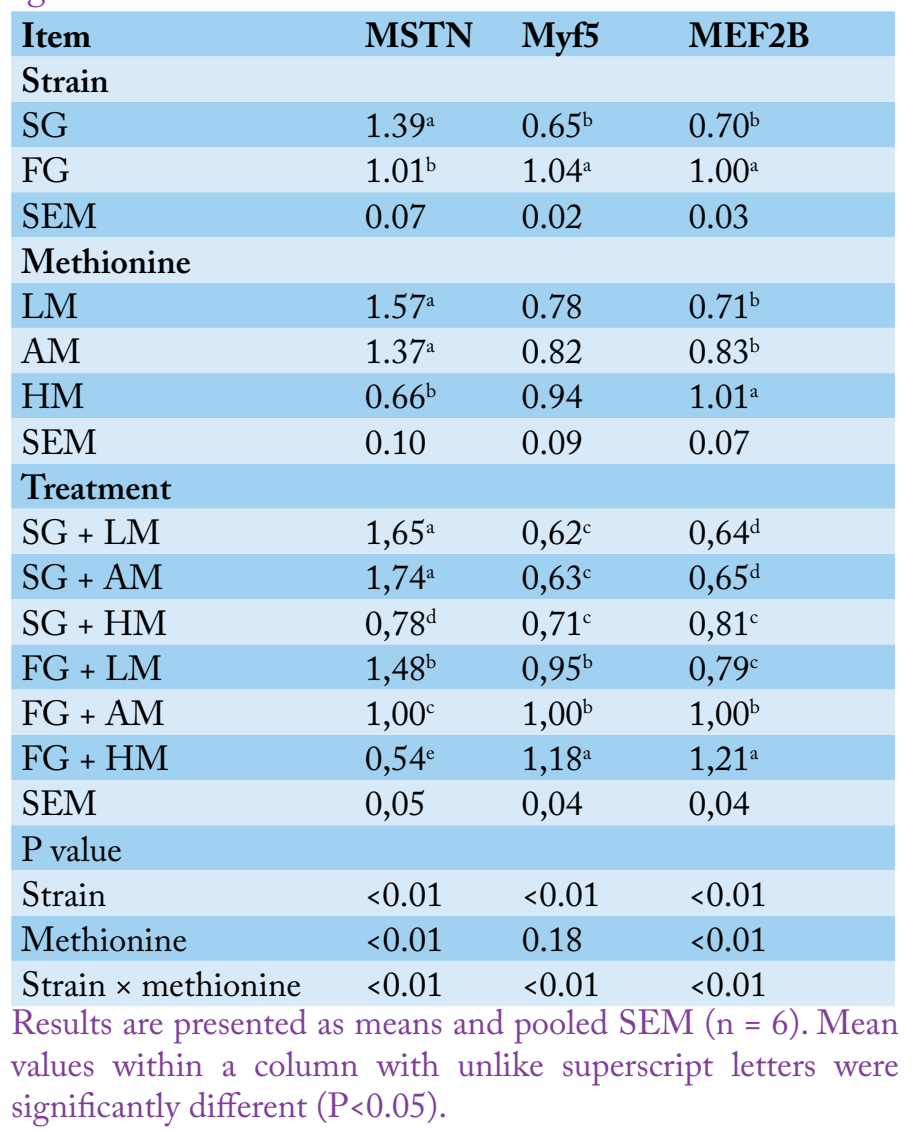

\section{DISCUSSIONS}

Results of this study generally show the different response between the two chicken strains to dietary SID methionine levels. For slow-growing strain, the body weight gain, FCR and PER were significantly $(\mathrm{P}<0.05)$ improved by feeding colored chicks with HM diets compared to AM and LM diets. These results were in agreement with findings of Pillai et al. (2006) who reported that weight gain and feed efficiency were significantly maximized with addition of methionine. Ahmed and Abbas (2011) also reported that broiler body weight gain, FCR and PER were significantly $(\mathrm{p}<0.05)$ improved by increasing dietary methionine compared to the methionine level according to NRC (1994) recommendation. The results of Hickling et al. (1990) also indicated that increasing the methionine level ( 0.43 vs. $0.38 \%$ Met) increased broiler weight gain and gain: feed (G:F) during 3 to 6 weeks of age. However, Wen et al. (2017) reported that using $-0.15 \%$ to $+0.1 \%$ methionine NRC (1994) was not affected body gain in the slow-growing broiler strain.

In the case of fast-growing strain, the BWG, FI, FCR and PER were not significantly $(\mathrm{P}>0.05)$ changed with the increasing of dietary SID methionine. This result was not in agreement with the finding of Zhai et al. (2012) who reported that methionine supplementation improved 
growth performance of the fast-growing broilers. Wen et al. (2017) also reported that comparing with using the diets with adequate or higher NRC (1994) methionine level increased body weight gain in the fast-growing broiler strain.

In spite the fact that the increasing dietary SID methionine level did not affect body weight gain in fastgrowing chickens, the breast muscle was improved and the abdominal fat retention was decreased when fed this strain with HM diet. The result of Ahmed and Abbas (2011) also indicated that dietary methionine higher than NRC (1994) increased breast muscle weight of broilers compared with the control. Meanwhile, Wen et al. (2017) found that breast muscle weight did not differ between the broilers fed the adequate and high NRC (1994) methionine diets. These conflicting results may due to the different dietary methionine, interaction between amino acids imbalance and response between chicken strains.

In this study, the HM diets decreased MSTN mRNA expression in breast muscle of chickens. This result agreed with the data of Liu et al. (2010), who found that methionine supplementation ( 0.61 vs. $0.21 \%$ during 4 to 8 weeks of age) downregulated myostatin mRNA expression in skeletal muscle of 55-d broilers. Increased breast muscle yield in fast-growing chicks in response to high SID methionine levels may be reflected by lower myostatin mRNA expression. The mRNA expressions of both Myf5 and MEF2B were greater in fast-growing chicks fed the $\mathrm{HM}$ diets. This result was similar to the finding of Wen et al. (2014), who indicated the unique role of these 2 genes distinct from other family members during myogenesis. The expression of Myf5 was not different between treatments in the case of slow-growing strain. Despite the greater MEF2B mRNA expression, the breast muscle yield was not improved when fed slow-growing chicks with HM diets. Therefore, this data implies that methionine effects on the formation of broiler breast muscle may be reflected by an upregulation in the mRNA expression of Myf5.

\section{CONCLUSIONS AND RECOMMENDATIONS}

The higher dietary SID methionine concentration than Evonik Recommendation (2010) improved growth performance in slow-growing colored chickens but had no effect on body gain in fast-growing strain. The increasing breast muscle yield and decreasing abdominal fat were observed in the fast-growing chicks fed high dietary SID methionine. The improvements in breast muscle of the fast-growing colored strain was reflected by increased mRNA expression of Myf5 and MEF2B but decreased expression of myostatin.

\section{ACKNOWLEDGMENTS}

This work was partially supported by Hue University under the Core Research Program, Grant No. NCM. DHH.2018.04.

\section{NOVELTY STATEMENT}

The study provides more information on the effects of SID methionine concentrations in diet on the growth performance, carcass yield and meat quality of two colored chicken groups that are commonly raised in the Central Vietnam. Besides, the modern molecular biological techniques were used to study the effects of dietary SID methionine on myogenic gene expression in these chicken groups.

\section{AUTHOR'S CONTRIBUTION}

Writing the manuscript, designing the experiment, data collection, data analyses: Ho Le Quynh Chau. Implementing the experiment, data collection: Than Thi Thanh Tra. Implementing the experiment, data collection: Duong Thi Huong. Giving comment on the manuscript: Du Thanh Hang. Implementing the experiment: Le Thi Thu Hang, Vo Thi Minh Tam. Giving comment on the manuscript, revising the manuscript: Dinh Van Dung. Giving comment on the manuscript: Ho Trung Thong.

\section{CONFLICT OF INTEREST}

The authors have declared no conflict of interest.

\section{REFERENCES}

Ahmed ME, Abbas TE (2011). Effects of dietary levels of methionine on broiler performance and carcass characteristics. Int. J. Poult. Sci., 10: 147-151. https://doi. org/10.3923/ijps.2011.147.151

Alami-Durante H, Cluzeaud M, Bazin D, Mazurais D, Zambonino-Infante JL (2011). Dietary cholecalciferol regulates the recruitment and growth of skeletal muscle fibers and the expressions of myogenic regulatory factors and the myosin heavy chain in European sea bass larvae. J. Nutr., 141: 2146-2151. https://doi.org/10.3945/jn.111.146118

Bouyeh M, Gevorgyan OK (2011). Infuence of excess lysine and methionine on cholesterol, fat and performance of broiler chicks. J. Anim. Vet. Adv., 10: 1546-1550. https://doi. org/10.3923/javaa.2011.1546.1550

Corzo A, Kidd M, Dozier W, Shack L, Burgess S (2006). Protein expression of pectoralis major muscle in chickens in response to dietary methionine status. Br. J. Nutr., 95: 703-708. https://doi.org/10.1079/BJN20051716

Drummond MJ, Glynn EL, Fry CS, Dhanani S, Volpi E, Rasmussen BB (2009). Essential amino acids increase microRNA-499, $-208 \mathrm{~b}$, and $-23 \mathrm{a}$ and downregulate myostatin and myocyte enhancer factor 2C mRNA expression in human skeletal muscle. J. Nutr., 139: 22792284. https://doi.org/10.3945/jn.109.112797 
Evonik (2010). AMINODat 4.0. In: Redshaw MS, Fickler J, Fontaine J, Heimbeck W, Hess V, and Reinmann I (Eds.), Evonik Industries, Evonik Degussa $\mathrm{GmbH}$, HanauWolfgang, Germany.

Hennebry A, Berry C, Siriett V, O'Callaghan P, Chau L, Watson T, Sharma M, Kambadur R (2009). Myostatin regulates fibertype composition of skeletal muscle by regulating MEF2 and MyoD gene expression. Am. J. Phys. Cell Phys., 296: C525-C534. https://doi.org/10.1152/ajpcell.00259.2007

Hickling D, Guenter W,Jackson ME (1990). The effects of dietary methionine and lysine on broiler chicken performance and breast meat yield. Can. J. Anim. Sci., 70: 673-678. https:// doi.org/10.4141/cjas90-079

Liu G, Zong K, Zhang L, Cao S (2010). Dietary methionine afect meat quality and myostatin gene exon 1 region methylation in skeletal muscle tissues of broilers. Agric. Sci. China, 9: 1338-1346. https://doi.org/10.1016/ S1671-2927(09)60224-8

Livak KJ, Schmittgen TD (2001). Analysis of relative gene expression data using real time quantitative $\mathrm{PCR}$ and the 2- $\Delta \Delta \mathrm{Ct}$ method. Methods, 25: 402-408. https://doi. org/10.1006/meth.2001.1262

NRC (1994). Nutrient requirements of poultry. $9^{\text {th }}$ Rev. ed. National Academy Press, Washington DC.

Nukreaw R, Bunchasak C, Markvichitr K, Choothesa A, Prasanpanich S, Loongyai W (2011). Effects of methionine supplementation in low-protein diets and subsequent refeeding on growth performance, liver and serum lipid profile, body composition and carcass quality of broiler chickens at 42 days of age. Jap. Poult. Sci., 48: 229-238. https://doi. org/10.2141/jpsa.010064

Pillai PB, Fanatico AC, Beers KW, Blair ME, Emmert JL (2006). Homocysteine remethylation in young broilers fed varying levels of methionine, choline and betaine. Poult. Sci., 85: 9095. https://doi.org/10.1093/ps/85.1.90

Ravindran V, Bryden WL (1999). Amino acid availability in poultry. In vitro and in vivo measurements. Aust. J. Agric. Res., 50(5): 889-908. https://doi.org/10.1071/AR98174

Schilling MW, Radhakrishnan V, Vizzier-Thaxton Y, Christensen K, Joseph P, Williams JB, Schmidt TB (2012). The effects of low atmosphere stunning and deboning time on broiler breast meat quality. Poult. Sci., 91(12): 3214-3222. https:// doi.org/10.3382/ps.2012-02266

Townley-Tilson WHD, Callis TE, Wang D (2010). MicroRNAs 1, 133, and 206: Critical factors of skeletal and cardiac muscle development, function, and disease. Int. J. Biochem. Cell Biol., 42: 1252-1255. https://doi.org/10.1016/j. biocel.2009.03.002

Wang Q, McPherron AC (2012). Myostatin inhibition induces muscle fiber hypertrophy prior to satellite cell activation. J. Phys., 590: 2151-2165. https://doi.org/10.1113/ jphysiol.2011.226001

Wen C, Chen X, Chen GY, Wu P, Chen YP, Zhou YM, Wang $\mathrm{T}$ (2014). Methionine improves breast muscle growth and alters myogenic gene expression in broilers. J. Anim. Sci., 92: 1068-1073. https://doi.org/10.2527/jas.2013-6485

Wen C, Jiang X, Ding L, Wang T, Zhou Y (2017). Effects of dietary methionine on breast muscle growth, mygene expression and IGF-I in fast- and slow-growing. Sci. Rep., 7: https://doi.org/10.1038/s41598-017-02142-z

Zhai W, Araujo LF, Burgess SC, Cooksey AM, Pendarvis K, Mercier Y, Corzo A (2012). Protein expression in pectoral skeletal muscle of chickens as influenced by dietary methionine. Poult. Sci., 91: 2548-2555. https://doi. org/10.3382/ps.2012-02213 